\title{
极区电离层 $\mathrm{O}^{+}$上行的时空分布: 平静期 FAST 卫星观测
}

\author{
党 戈 马淑英 ${ }^{*}$ 周云良
}

(武汉大学电子信息学院, 教育部地球空间环境与大地测量重点实验室, 武汉 430079. * 联系人, E-mail: syma@whu.edu.cn)

\begin{abstract}
摘要 分析FAST卫星能量离子测量数据, 首次得到 2000 4200 $\mathrm{km}$ 高度范围平静期电离层 $\mathrm{O}^{+}$上行的时 空分布图像. 结果表明, 磁正午前的极尖/极隙区是低能 $\mathrm{O}^{+}$上行发生最频繁的区域; 黎明前等离子体对 流的低纬边界区域是高能 $\mathrm{O}^{+}$上行发生率最高区. 无论能量高低, 在平静极光椭圆边界之外的较低纬度 宽阔时区上存在较频繁的 $\mathrm{O}^{+}$上行. 午夜前 21:00 22:00 MLT极光椭圆带的上行 $\mathrm{O}^{+}$携有较强的能通量, 其中高能 $\mathrm{O}^{+}$在靠近极盖边界处上行能通量特别强. 在所分析的 $2000 \sim 4200 \mathrm{~km}$ 高度范围, 上行 $\mathrm{O}^{+}$离子雉 发生率显著高于离子束; 离子束在 $3000 \mathrm{~km}$ 高度以下极少出现, 而离子雉较均匀地在各高度都有发生.
\end{abstract}

关键词 氧离子上行 极区电离层 电离层-磁层耦合 卫星观测

地球磁层最基本的问题之一是其等离子体的来 源. 由于较早的空间探测仪器只对高能粒子敏感, 且 无法分辨不同质量成分，使得人们难以区分源于太 阳的和源于地球的磁层粒子成分, 曾一度认为太阳 风是磁层等离子体的最主要来源. 1972 年Shelley等人 [1首次发现在磁层中存在源于电离层的 $\mathrm{O}^{+}$离子, 此后 人们对电离层离子的上行源区及其在地球空间中获 能与传输过程等问题展开了广泛的研究. 近 30 多年 来, 随着能量粒子探测仪器和卫星电位主动控制技 术的发展, 卫星观测证实, 在极区电离层中存在着多 种离子整体外流和获能过程, 它们使电离层成为磁 层等离子体的重要来源(文献 [2 4]及其所引文献).

在极区既存在着太阳风和地球磁层粒子不断沉 降进入电离层/高层大气, 也存在着电离层等离子体 持续不断向外流出; 外流离子的成分有较轻的热 (thermal) 离子 $\left(\right.$ 如 $\mathrm{H}^{+}, \mathrm{He}^{+}$), 也有高能的 (energetic) 轻 离子和重离子 $\left(\right.$ 如 $\mathrm{O}^{+}, \mathrm{NO}^{+}, \mathrm{O}_{2}^{+}$等). 较重的离子 $\mathrm{O}^{+}$(其 质/荷比 16)要逃逸出电离层必需获得至少 $10 \mathrm{eV}$ 的 能量. $\mathrm{H}^{+}$和 $\mathrm{O}^{+}$离子外流特征的一个重要差别是后者 与地磁活动性的强烈相关, 磁暴期间磁层中 $\mathrm{O}^{+}$离子 成分极大地增强, 有时会成为暴时环电流的主要成 分 ${ }^{[5]}$, 特别是大磁暴期间. 这是近 10 余年来磁暴与 磁层动力学研究的一个突破性进展. 环电流中这些 源自电离层的 $\mathrm{O}^{+}$在源区的分布是怎样的, 驱使它们 进入磁层, 进入特定区域环电流的加速/获能过程是
怎样的, 或者它们是否已经存在于磁层中的某个区 域, 至今未完全搞清楚, 是地球空间暴研究中的重要 课题. 而要研究暴时电离层 $\mathrm{O}^{+}$离子上行源区的特征, 需要对平静时背景上行有清楚的了解. 最近, 我国学 者提出了“磁层亚暴锋面触发模型”的新思想 ${ }^{1)}$, 认为 电离层重离子上行在锋面形成, 触发亚暴过程中起 着关键性的重要作用. 平静时和亚暴前电离层 $\mathrm{O}^{+}$离 子上行特征的研究对于验证和发展这一新思想, 揭 开亚暴触发之谜具有重要的意义.

离子上行过程可分为两类: 整体离子上行(bulk ion upflows)和离子获能过程(ion energization processes $)^{[4]}$. 在整体离子上行中, 所有离子获得一个整 体上行速度, 其能量最高达几个 $\mathrm{eV}$, 具体包括沿开放 磁力线的主要由 $\mathrm{H}^{+}$构成的极风(polar wind)以及 $\mathrm{O}^{+}$离 子占主导的极光带高速整体上行离子流. 在离子获 能过程中, 只有一部分上行离子获能, 成为超热 (superthermal)或能量更高的离子; 比如离子束, 离子 雉, 横向加速离子和上涌离子流等. 发生在不同位置 的离子上行事件有着不同的形态特征并与不同的加 速机制相联系,利用统计方法分析离子上行事件时空 分布与变化特征, 对深入认识上行过程有重要意义.

对于大约 $2000 \mathrm{~km}$ 高度以上源于电离层的 $\mathrm{O}^{+}$离 子上行的时空分布规律, Klumpar ${ }^{[6]}$ 曾利用ISIS-1 和 ISIS-2 卫星数据, 研究了大约 1000 3000 km高度上 横向加速离子(未区分其质量成分)的出现与地方时 
和季节以及高度的关系. Gorney等人 ${ }^{[7]}$ 利用 $S 3-3$ 卫星 分析了约 250 8000 km高度范围离子束和离子雉随 高度和磁地方时的变化特征, 但使用的是静电分析 仪测量数据, 未区分离子成分. Yau等人 ${ }^{[8,9]}$ 利用DE-1 卫星数据研究了 $8000 \sim 23000 \mathrm{~km}$ 高度范围 $\mathrm{O}^{+}$和 $\mathrm{H}^{+}$离 子上行发生率受太阳活动水平和季节的影响, 上行 通量随纬度和磁地方时的分布及其对地磁活动水平 的依赖. Kondo等人 ${ }^{[10]}$ 利用DE-1 卫星数据得到了该 卫星覆盖的高度范围里 $\mathrm{O}^{+}$和 $\mathrm{H}^{+}$离子束和离子雉的时 空分布. Thelin等人 [11]利用Viking卫星分析了 5000 $13500 \mathrm{~km}$ 高度极尖/极隙区上行离子束和离子雉事件 发生的时空分布. IMAGE卫星低能中性原子成像测 量为研究电离层 $\mathrm{O}^{+}$离子上行提供了新的手段 ${ }^{[12]}$. 美 国宇航局 1996 年发射升空的极光快速抓拍(FAST)卫 星 ${ }^{[13]}$, 其高度覆盖约 400 4000 km, 远地点位于通常 的极光粒子加速区下边界附近，该卫星上的多种粒 子探测器为研究电离层离子上行提供了宝贵数据, 至今已有不少这方面的研究, 如文献 [14 20]. 但是 这些研究主要是对某些特殊上行事件或较短时间覆 盖数据的分析, 或者侧重某几种上行机制的研究, 对 于利用FAST覆盖多年数据进行平静时电离层 $\mathrm{O}^{+}$上行 特征及其磁暴期间变化的统计分析研究尚未见报道. 本文即利用极光快速抓拍(FAST)卫星离子质谱仪 ${ }^{[21]}$ 数据, 研究平静时极区电离层 $\mathrm{O}^{+}$上行源区域特征, 为 进一步研究其暴时变化提供必需的背景参照.

本文首先介绍数据及其分析处理, 包括来源和 选取, $\mathrm{O}^{+}$离子上行事件的确定及其分析处理方法; 然 后介绍统计分析结果, 作简要讨论; 最后对本文主要 发现与结果进行总结.

\section{1 数据及其分析处理}

\section{1 数据的来源与选取}

本文使用美国宇航局极光快速抓拍(Fast Auroral SnapshoT, 缩写为FAST)卫星 [22]粒子测量数据. FAST 卫星于 1996 年 8 月发射升空, 椭圆轨道, 倾角 $83^{\circ}$, 近地点高度 $350 \mathrm{~km}$, 远地点 $4175 \mathrm{~km}$, 开普勒周期约 133 分钟; 轨道面进动为每 8.1 个月覆盖全部地方时. FAST卫星上的测量仪器通常每从较低纬度进入极光 区才开机运行, 离开极光区向低纬度飞行时仪器关闭.

卫星上装有多种场与粒子探测仪器, 本研究主 要使用能量-投郑角-质谱分析仪 (Time-of-flight Energy Angle Mass Spectrograph, 缩写为TEAMS $)^{[211}$ 测
量数据. 该质谱仪测量几种主要离子(包括 $\mathrm{H}^{+}, \mathrm{He}^{++}$, $\mathrm{He}^{+}$和 $\mathrm{O}^{+}$等)的三维速度分布, 对于 $\mathrm{H}^{+}$和 $\mathrm{O}^{+}$, 最快可以 在 $1 / 2$ 个卫星自旋周期 $(2.5 \mathrm{~s})$ 内得到离子通量随投郑 角和能量的分布; 能够瞬时接收来自 $0^{\circ} \sim 360^{\circ}$ 方位角 的离子, 分为 16 个方位像素, 分辨率为 $22.5^{\circ}$; 能量范 围 $1.2 \mathrm{eV} \sim 12 \mathrm{keV}$, 能量间隔 $(\Delta E / E)$ 约为 0.13 , 共 48 个 能量等级. 卫星表面电位会造成能量较低离子测量的 不准确, 在无法获得卫星电位数据以进行修正的情况 下, 我们舍弃了能量小于 $5 \mathrm{eV}$ 的数据; 为了消除卫星 在近地点附近高速飞行时撞击效应 (Ram Effect)的影 响, 把卫星高度低于 $2000 \mathrm{~km}$ 的数据也剔除掉.

卫星上还装有离子静电分析仪 IESA (Ion ElectroStatic Analyzer), 提供离子(不区分种类)三维速度分 布. IESA 仪器性能比 TEAMS 稳定; 随着时间的推移, TEAMS 的灵敏度有所降低, 而且有时在某个测量通 道上工作不正常, 出现反常大的通量; 所以我们用 IESA 测量数据在某种程度上判断 TEAMS 数据的合 理性. 将 IESA 测量到的总的(不区分离子成分)离子 微分能通量作为参考值, 把 TEAMS 同时测得的 $\mathrm{O}^{+}$ 微分能通量与此参考值作比较, 考虑到可能的正常 测量误差, 如果 TEAMS 测得的 $\mathrm{O}^{+}$微分能通量大于参 考值的 10 倍, 那么这个时刻 TEAMS 测量数据将从统 计的数据库里剔除掉. 图 1 给出了一个选进统计数据 库的能通量角谱 (a)和一个被剔除的能通量角谱(b)的 例子; 在被剔除的事例中, 在 $180^{\circ}$ 投郑角通道上 $\mathrm{O}^{+}$ 微分能通量 $\left(F_{\text {diff }}\right)$ 比同时的 IESA 测量到的总离子微 分能通量高一个数量级以上.

由于FAST卫星轨道是椭圆, 高度在较大范围内 变化, 为了对上行 $\mathrm{O}^{+}$离子通量随纬度和地方时的分 布特征进行分析, 我们把卫星在不同高度测量得到 的微分能通量值和相应的坐标沿磁力线映射到同一参 考高度 $1000 \mathrm{~km}$, 映射时所使用的磁场模型是IGRF2003 外加Tsyganenko-2001 模型 ${ }^{[23,24]}$.

关于平静期数据的选择, 从 1997 2003 这 7 年 FAST/TEAMS 有合理观测数据的日子中挑选出平静 日子的数据; 平静日子的标准是: 处在德国波茨坦地 学研究中心 GFZ-Potsdam 网站公布的每月相对最平 静的 $10 \mathrm{~d}$ 之中, 并且当天 8 个 $K_{\mathrm{p}}$ 值都小于 3 . 需要说 明, 选取的 FAST/TEAMS 数据中不包含 2001 年 11 月 12 月两个月的数据, 因为此间 TEAMS 仪器出现 故障. 另外本文只选用北半球的观测数据, 纬度范围 

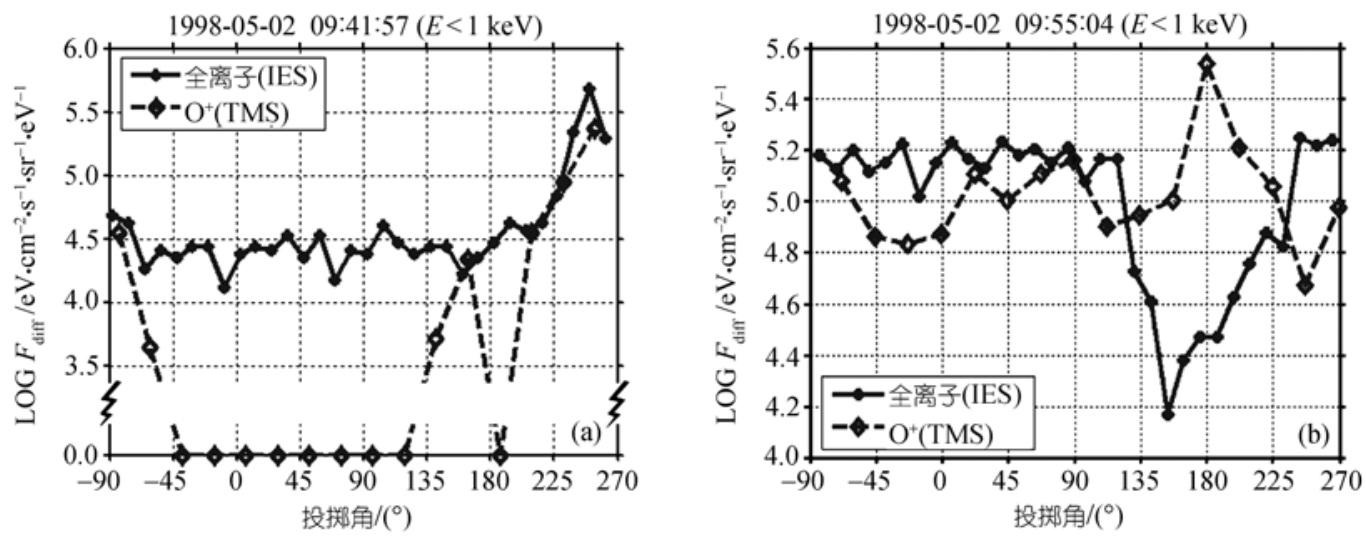

图 1

(a) 选进统计数据库的 $\mathrm{O}^{+}$微分能通量谱示例; (b) 被剔除的 $\mathrm{O}^{+}$微分能通量角谱示例. 虚线表示 TEAMS 测量得到的 $\mathrm{O}^{+}$微分能通量, 实线表示 IESA 测量得到的总的离子微分能通量

限于 $60^{\circ} \sim 80^{\circ}$ MLat. 这样选择出平静期合理的 TEAMS 测量数据点共约 37 万个, 跨越 1997 2003 年半个多 太阳黑子周期, 涵盖 $407 \mathrm{~d}$. 对这些数据点所对应的 卫星轨道的磁纬、磁地方时及高度覆盖与分布情况, 以及行星际磁场 $B_{y}$ 分量取值(正或负)分布, 进行了统 计. 图 2(a)给出 FAST 卫星轨道的磁纬(MLat)和磁地 方时(MLT)覆盖情况. 可以看到, 所用数据对 $60^{\circ} \sim 80^{\circ}$ 磁纬和所有磁地方时的覆盖都非常好. 图 2(b)给出合 理的 FAST/TEAMS 测量数据点数目 $\left(N_{\mathrm{M}}\right)$ 随高度的分 布情况; 可以看到, 约 $51 \%$ 的数据在 $3500 \sim 4200 \mathrm{~km}$ 高度, 2000 3500 km 高度范围数据约占 $49 \%$. 另外, IMF $B_{y}$ 为负的数据点占 $47 \%$, 为正的数据点占 $53 \%$, 大体持平.

\section{2 数据的处理与统计分析方法}

首先我们要给出“来自较低高度电离层的 $\mathrm{O}^{+}$离 子上行事件”(后文中简称 $\mathrm{O}^{+}$离子上行事件)的定义. 在所研究的极光区上空 2000 4000 $\mathrm{km}$ 高度区域, 存
在着来自较低高度电离层的上行 $\mathrm{O}^{+}$离子, 同时也大 量存在着从磁层, 主要是等离子体片区, 沿磁力线沉 降下来又从卫星高度以下的磁镜点反射上行的 $\mathrm{O}^{+}$离 子, 以及投郑角在损失雉内而沉降进入大气层的 $\mathrm{O}^{+}$ 离子. 如果仅仅根据在投掷角 $\alpha$ 为 $90^{\circ} \sim 270^{\circ}$ 上有显著 的通量值而定义其为上行事件, 那么就混杂了较大 量的从磁镜点反射上行的原已存在于磁层中的 $\mathrm{O}^{+}$粒 子. 为了消除这一成分, 我们计算净通量 $F_{\text {net }}$,

$$
F_{\text {net }}=F_{\text {up }}-F_{\text {down }} \text {, }
$$

其中

$$
\begin{aligned}
& F_{\text {up }}=\left|\int_{\frac{\pi}{2}}^{\frac{3}{2} \pi} f(\alpha) \cos \alpha d \alpha\right|, \\
& F_{\text {down }}=\left|\int_{-\frac{\pi}{2}}^{\frac{\pi}{2}} f(\alpha) \cos \alpha d \alpha\right| .
\end{aligned}
$$
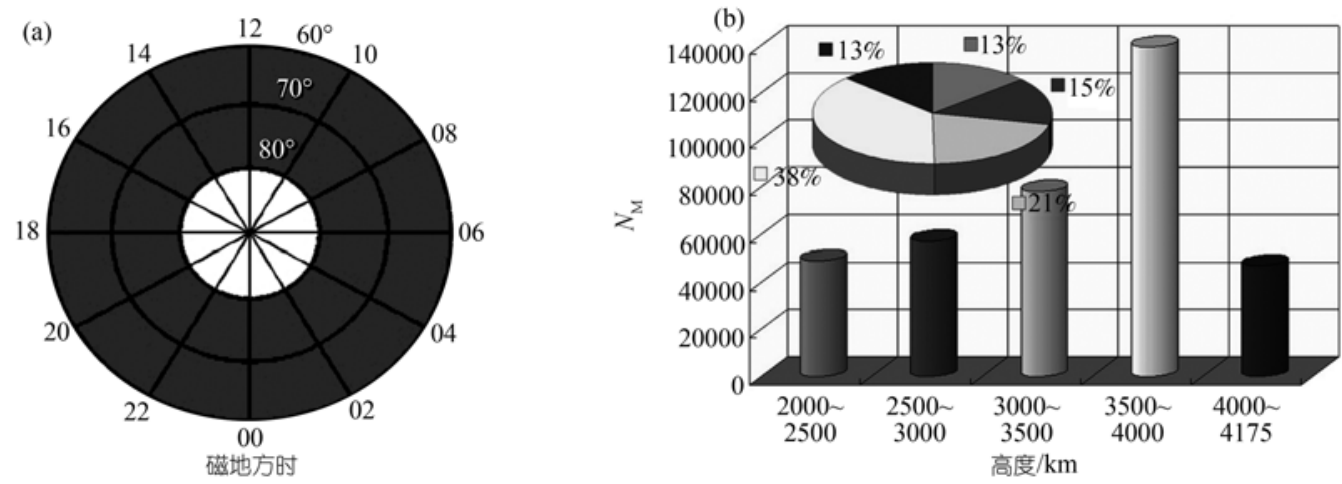

图 2

(a) FAST 卫星轨道的磁纬和磁地方时覆盖; (b) 所用数据点总数随高度的分布 
在镜点反射并往复弹跳的离子, 其投搠角分布可以 近似认为各向同性, 这样相减之后就被消除了; 但是 这样得到的净通量, 从来自电离层的上行离子通量 中扣除了投郑角在损失雉内的沉降进入大气层的 $\mathrm{O}^{+}$ 离子. 考虑到与 $\mathrm{O}^{+}$离子上行同时存在的 $\mathrm{O}^{+}$沉降一般 很弱, 而且沉降至大气层的 $\mathrm{O}^{+}$离子通量目前难以估 计而加以修正, 所以将上述净通量取值大于零作为 存在 $\mathrm{O}^{+}$离子上行的必要条件. 再考虑可能的噪音误 差, 我们计算平静期净通量的标准差 $\sigma_{\mathrm{F}}$ 如下:

$$
\sigma_{\mathrm{F}}=\left(\frac{1}{n-1} \sum\left[F_{\text {net }}-\mu_{\mathrm{F}}\right]^{2}\right)^{1 / 2},
$$

其中

$$
\mu_{\mathrm{F}}=\frac{1}{\mathrm{n}} \sum F_{\text {net }},
$$

式中, $n$ 表示总的采样点个数. 净通量至少大于 $3 \sigma_{\mathrm{F}}$, 才将其视为一个 $\mathrm{O}^{+}$离子上行事件. 根据表 1 给出的平 静时低能 $(E<1 \mathrm{keV})$ 和高能 $(E>1 \mathrm{keV})$ 背景 $\mathrm{O}^{+}$净通量的 标准差, 我们统一地将此限值取为 $10^{4} \mathrm{eV} /\left(\mathrm{cm}^{2} \cdot \mathrm{s} \cdot \mathrm{eV}\right)$. 因此, 在本文中, 一个 $\mathrm{O}^{+}$上行事件发生是这样定义 的: 当某个时刻 $\mathrm{O}^{+}$的净通量大于 $10^{4} \mathrm{eV} /\left(\mathrm{cm}^{2} \cdot \mathrm{s} \cdot \mathrm{eV}\right)$ 时, 记为一个上行事件; 又因为此时我们没有区分离 子雉、离子束、离子整体上行以及离子上涌等, 所以 称之为一个总的 $\mathrm{O}^{+}$上行事件.
表 1 背景 $\mathrm{O}^{+}$净通量的均值 $\mu_{\mathrm{F}}$ 和标准差 $\sigma_{\mathrm{F}}$ (单位: $\mathrm{eV} /\left(\mathrm{cm}^{2} \cdot \mathrm{s} \cdot \mathrm{eV}\right)$

\begin{tabular}{ccc}
\hline & $\mu_{\mathrm{F}}$ & $\sigma_{\mathrm{F}}$ \\
\hline$E<1 \mathrm{keV}$ & 377.18 & 2889.1 \\
$E>1 \mathrm{keV}$ & -5.6122 & 2457.5 \\
\hline
\end{tabular}

从总的 $\mathrm{O}^{+}$上行事件中再挑选出上行离子束和离 子雉事件. 在北半球, 离子束的特点是在 $180^{\circ}$ 投掷角 方向上能通量有锐的最大值, 离子雉最大能通量所在 的投郑角与磁场方向有一定夹角. 但由于受 TEAMS 仪器投掷角分辨率的影响, 离子束和离子雉的投掷 角范围如表 2 所示. 确定 $\mathrm{O}^{+}$上行离子束/雉事件的标 准是: 对于上面所挑选出来的总的 $\mathrm{O}^{+}$上行事件, 如 果它的微分能通量峰值所在的投郑角落在表 2 给出 的离子束/雉投郑角范围内, 且其值大于 $10^{5} \mathrm{eV} /\left(\mathrm{cm}^{2}\right.$. $\mathrm{s} \cdot \mathrm{sr} \cdot \mathrm{eV})($ 参照文献 $[25,26])$ 并大于该能通量谱平均值 的 1.5 倍, 我们则视其为上行离子束/雉事件.

表 2 离子束和离子雉的投郑角范围(北半球)

\begin{tabular}{cc}
\hline & 投郑角 \\
\hline 离子束 & $157.5^{\circ} \leq \alpha \leq 202.5^{\circ}$ \\
离子锥 & $112.5^{\circ} \leq \alpha<157.5^{\circ}$ 或 $202.5^{\circ}<\alpha \leq 247.5^{\circ}$ \\
\hline
\end{tabular}

图 3 给出 FAST 卫星观测到的典型的上行 $\mathrm{O}^{+}$离 子束与离子雉事例. (a)

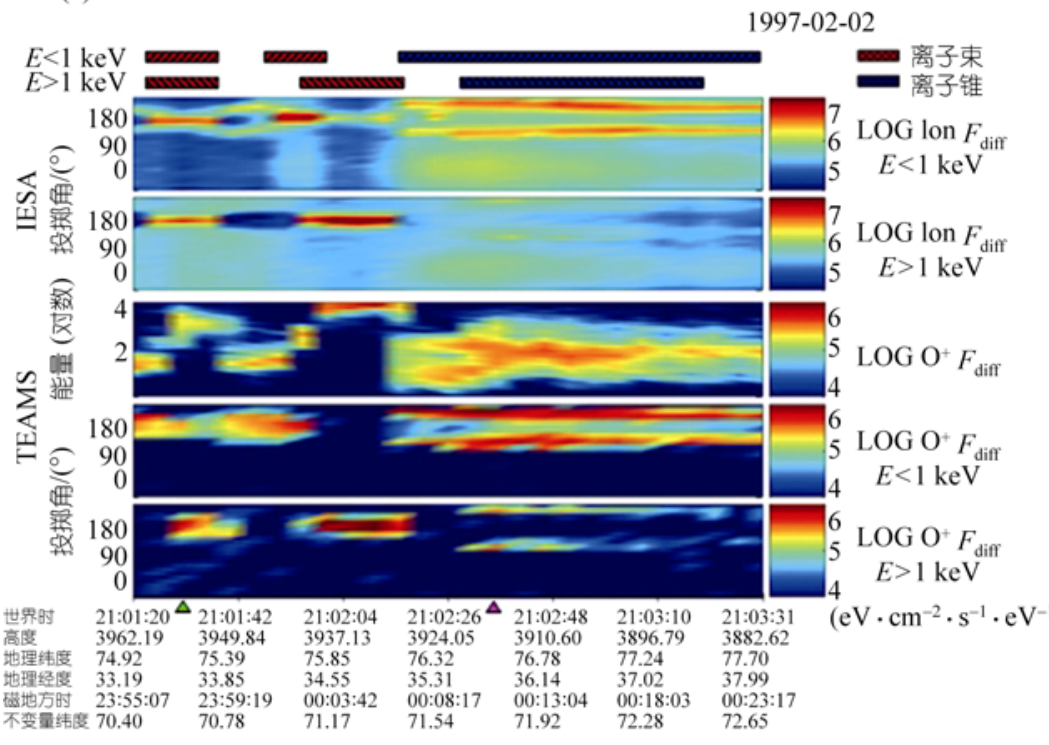

图 $3 \mathrm{O}^{+}$上行离子束/雉典型事例 (b) FAST轨道1789

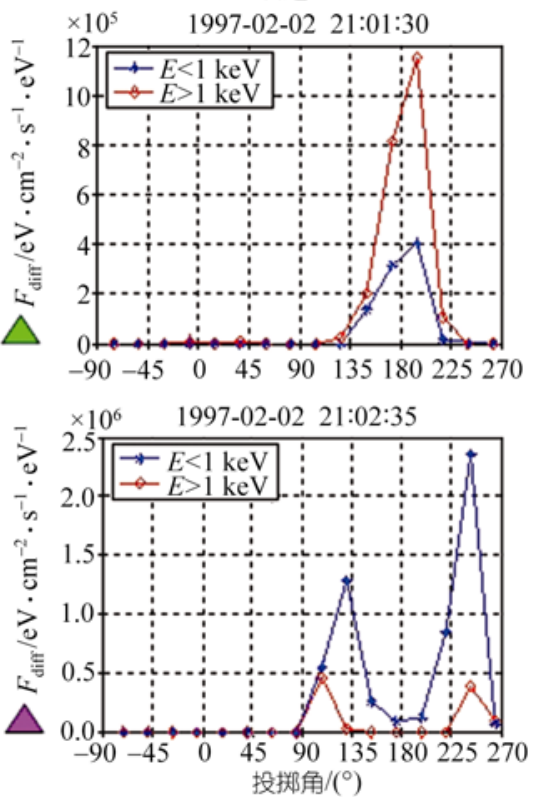

(a) 随时间变化的离子微分能通量谱, 从上至下依次是: IESA 低能离子角谱, IESA 高能离子角谱, TEAMS 氧离子能谱, TEAMS 低能 $\mathrm{O}^{+}$离子角谱, TEAMS 高能 $\mathrm{O}^{+}$离子角谱; (b) 在(a)中用绿色和粉色三角形所示两个典型时刻, $\mathrm{O}^{+}$微分能通量随投郑角的变化, 蓝线对应能量低于 $1 \mathrm{keV}$, 红线对 
所作统计分析, 分别对低能 $(E<1 \mathrm{keV})$ 和高能 $(E$ $>1 \mathrm{keV}$ )的上行离子进行. 为了研究上行 $\mathrm{O}^{+}$离子特征 随地磁纬度 (MLat) 和磁地方时 (MLT) 的分布, 对 MLat $\times$ MLT 坐标平面进行网格划分. 地磁纬度覆盖 $60^{\circ} \sim 80^{\circ}$, 磁地方时覆盖 $0 \sim 24 \mathrm{~h}$. 纬度和时间间隔选 取的准则是, 既要尽可能的把不同磁纬和磁地方时 上行 $\mathrm{O}^{+}$离子的特征差异表现出来, 同时也要保证在 每个网格里的数据点个数具有统计意义. 对于平静 时期划分为 240 个网格, MLat 分为 10 组, 间隔取 $2^{\circ}$, MLT 分为 24 组, 间隔取 $1 \mathrm{~h}$, 这样划分后每个网格的 数据点数都在 400 个以上. 另外, 为了分析 $\mathrm{O}^{+}$离子上 行分布的半球不对称性, 还做了另一种分组, 即对纬 度不做分隔, 只将磁地方时分为向日半球 (06-12$18 \mathrm{MLT})$ 和背日半球(18-00-06)MLT, 以及晨侧半球 (00-06-12MLT)和昏侧半球(12-18-00MLT).

对于上行离子束/雉, 因事件个数较少, 如果按 上面的方法划分网格, 分配到部分网格里的数据点 就很少, 不具有统计意义, 因此只考虑 $\mathrm{O}^{+}$上行离子 束/雉特征(事件发生率, 平均能通量)随磁地方时的 分布, 以及随高度的变化, 其中磁地方时间隔取 $3 \mathrm{~h}$, 高度间隔取 $500 \mathrm{~km}$.

上行事件及其分组确定后, 即可按照下式计算 在每一组内总的上行事件发生率 $P_{\mathrm{up}}$ :

$$
P_{\text {up }}=N_{\text {up }} / N_{\text {all }},
$$

其中, $N_{\text {up }}$ 表示每一组内总的 $\mathrm{O}^{+}$离子上行事件个数, $N_{\text {all }}$ 表示该组所有合理的数据采样点个数. 相应地, 每组上行 $\mathrm{O}^{+}$平均能通量 $\overline{F_{\text {up }}}$ 为:

$$
\overline{F_{\text {up }}}=\frac{\sum_{1}^{N_{\text {up }}} F_{\text {net }}}{N_{\text {up }}},
$$

其中, $\sum_{1}^{N_{\text {up }}} F_{\text {net }}$ 表示该组中所有上行事件的净能通量 之和, $N_{\text {up }}$ 表示该组中上行事件个数.

类似地, 对于给定的能级, 在每个 MLT 扇区上 行离子束和离子雉事件的发生率 $P_{\mathrm{b}}$ 和 $P_{\mathrm{c}}$ 分别为:

$$
\begin{aligned}
& P_{\mathrm{b}}=N_{\mathrm{b}} / N_{\mathrm{all}}, \\
& P_{\mathrm{c}}=N_{\mathrm{c}} / N_{\mathrm{all}},
\end{aligned}
$$

其中, $N_{\mathrm{b}}$ 和 $N_{\mathrm{c}}$ 分别表示在给定的能级, 该 MLT 扇区 上行离子束或离子雉事件个数(含所有纬度和高度), $N_{\text {all }}$ 表示该 MLT 扇区(含所有纬度和高度)所有合理的 数据采样点个数; 在考虑随高度变化时, 数据点与事
件个数对所有纬度和磁地方时求和. 每个 MLT 扇区 上行离子束/雉事件的平均能通量 $\overline{F_{\mathrm{b}}}$ 和 $\overline{F_{\mathrm{c}}}$ 定义为:

$$
\begin{aligned}
& \overline{F_{\mathrm{b}}}=\sum_{N_{\mathrm{b}}} F_{\mathrm{b}} / N_{\mathrm{b}}, \\
& \overline{F_{\mathrm{c}}}=\sum_{N_{\mathrm{c}}} F_{\mathrm{c}} / N_{\mathrm{c}},
\end{aligned}
$$

其中, $\sum_{N_{\mathrm{b}}} F_{\mathrm{b}}$ 和 $\sum_{N_{\mathrm{c}}} F_{\mathrm{c}}$ 分别表示每个 MLT 扇区上行离 子束或离子雉净能通量之和, $N_{\mathrm{b}}$ 和 $N_{\mathrm{c}}$ 分别表示每个 MLT 扇区相应上行离子束或离子雉事件个数.

本文数据处理的流程可概括如图 4 .

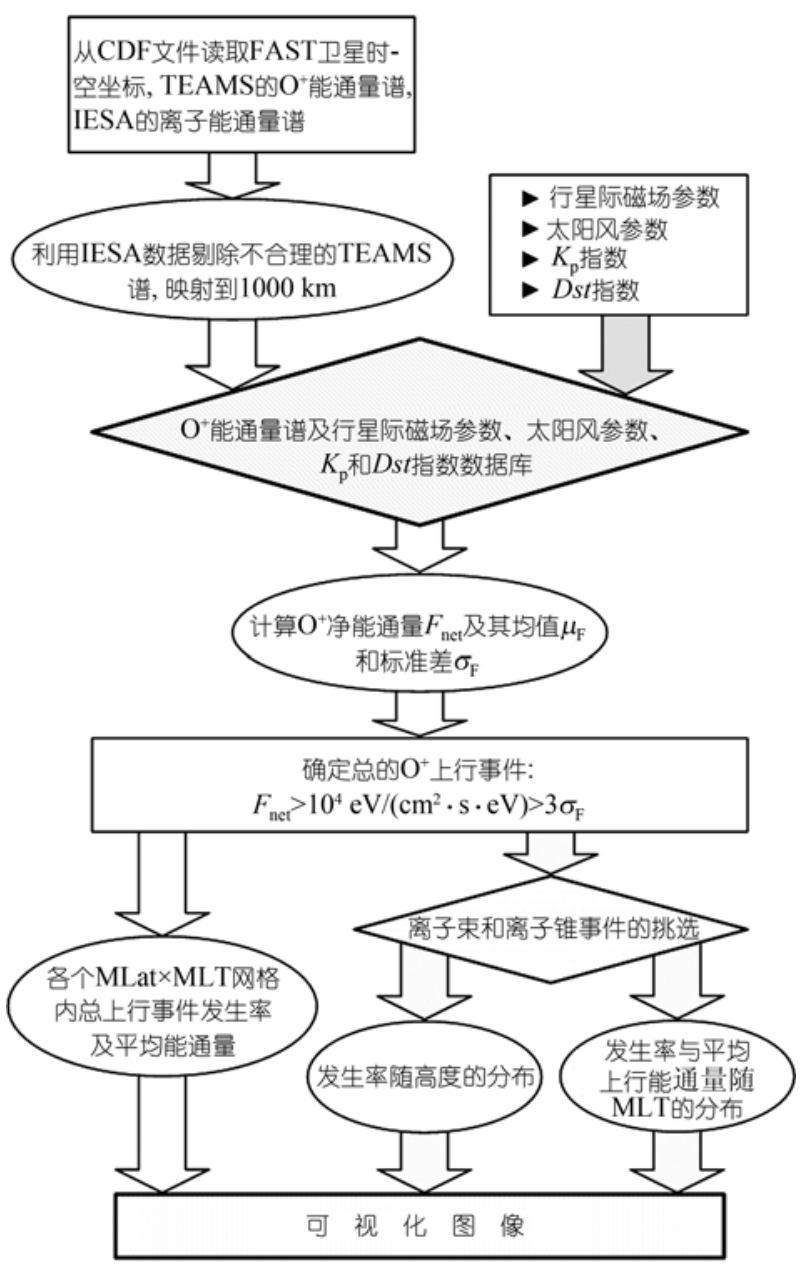

图 4 数据处理流程

这里需要指出, 我们选用的平静期数据包括太 阳活动上升相、高年、和下降相, 包括不同的季节, 对 应不同的行星际条件. 本文不研究电离层氧离子上 行对季节和太阳活动水平的响应, 对行星际条件的 依赖也另外研究; 只探讨地磁平静时氧离子上行的 1 平均统计规律, 5 为进 2 步研究磁暴期间的特征提供 
参照背景. 所以流程中将 $K_{\mathrm{p}}$ 指数和 $D s t$ 指数都加入数 据库; 有关暴时特征的分析结果将另文发表.

\section{2 分析结果}

经过上述步骤的分析处理和计算, 得到平静期 对于不同能量等级 $(E<1 \mathrm{keV}, E>1 \mathrm{keV})$, 总的 $\mathrm{O}^{+}$上 行事件的发生率和平均能通量随磁纬和磁地方时的 二维分布, 以及离子束和离子雉事件发生率和平均 能通量分别随磁地方时和随高度的分布, 其特征详 述如下.

\section{1 总的 $\mathrm{O}^{+}$上行}

(i ) 总的 $\mathrm{O}^{+}$上行事件发生率. 图 5 给出了平静 期低能 $(E<1 \mathrm{keV})$ 和高能 $(E>1 \mathrm{keV}) \mathrm{O}^{+}$总的上行事 件(不区分整体上行、上涌、横向加速、离子雉和离 子束)发生率 $\left(P_{\mathrm{up}}\right)$ 随磁纬和磁地方时的分布图. 各个 图中左上角的小极图是各个网格中数据点总数 $\left(N_{\mathrm{M}}\right)$.

从图 5 可以看到, 平静时低能 $\mathrm{O}^{+}$上行事件主要 发生在磁正午前后 08:00 13:00 MLT 扇区, 72。 78 MLat 之间, 发生率最大值 0.37; 在磁午夜前 20:00 23:00 MLT 较低纬度 $\left(65^{\circ}\right.$ 以下)也有较显著的上行发 生率. 高能 $\mathrm{O}^{+}$上行事件发生率总体上小于能量较低 的 $\mathrm{O}^{+}$, 主要发生在黎明前后 $4 \mathrm{~h}$ 之内的扇区, 峰值约 0.32 出现在黎明前 04:00 05:00 MLT 之间比较低的 纬度上; 磁午夜前较低纬度 $\left(65^{\circ}\right.$ 以下)也有尚为显著 (20\%以上)的上行发生率.

还可以看出, 对于低能部分, 向天半球上行发生 率略高于夜晚半球，黎明侧半球发生率明显高于黄 昏半球; 而对于高能部分, 夜晚半球上行发生率显著 高于白天半球, 晨侧高于昏侧的不对称性比低能情 况显著, 参见表 3.

(ii)上行事件能通量. 总的 $\mathrm{O}^{+}$上行事件的平 均能通量 $\left(\overline{F_{\text {up }}}\right)$, 我们称之为上行事件平均强度, 其 随磁纬和磁地方时的变化如图 6 所示.

可以看到, 无论是低能还是高能离子, 强度较大

表 3 平静期总的 $\mathrm{O}^{+}$上行事件发生率的半球分布

\begin{tabular}{cccc}
\hline & \multicolumn{2}{c}{ 发生率 $(\%)$} \\
\cline { 3 - 4 } & & 低能 $\mathrm{O}^{+}(E<1 \mathrm{keV})$ & 高能 $\mathrm{O}^{+}(E>1 \mathrm{keV})$ \\
\hline \multirow{2}{*}{ 日/夜半球 } & 向日 & 17.1 & 12.2 \\
& 背日 & 16.5 & 16.2 \\
\multirow{2}{*}{ 晨/昏半球 } & 黎明 & 17.8 & 18.3 \\
& 黄昏 & 15.7 & 11.2 \\
\hline
\end{tabular}

的上行都是出现在午夜前扇区一个比较宽的区域里, 磁地方时范围从 20:00 MLT 到 00:00 MLT, 纬度范 围从 $64^{\circ} \mathrm{MLat}$ 到 $76^{\circ} \mathrm{MLat}$; 最大的上行强度发生在 21:00 22:00 MLT 之间, 而且低能离子在 $66^{\circ} \sim 72^{\circ}$ 和 $74^{\circ} \sim 78^{\circ} \mathrm{MLat}$ 出现两个强上行区, 被较弱上行强度纬 度带隔开, 高能离子也出现类似情况, 不过它在 $74^{\circ} \sim 76^{\circ} \mathrm{MLat}$ 出现非常显著的最大上行能通量. 另 外, 低能离子在极尖/极隙区附近 $09: 00$ 13:00 MLT, $76^{\circ}$ MLat 附近) 也有较大的上行强度, 而高能离子 没有.

\section{2 上行 $\mathrm{O}^{+}$离子束和离子雉}

(i) 发生率随高度的变化. 图 7 给出上行 $\mathrm{O}^{+}$ 离子束和离子雉事件发生率随高度的分布. 比较图 7(a)和(b)两列可以看到, 在所分析的 2000 4200 km 高度, $\mathrm{O}^{+}$离子雉事件发生率明显高于离子束事件(注 意, 雉和束两图中纵坐标的标度不同).

对于离子束事件, 发生的高度主要分布在 3500 $\mathrm{km}$ 高度以上, 在 $3000 \mathrm{~km}$ 以下很少发现离子束事件. 这一结果和Gorney等人 ${ }^{[7]}$ 分析S3-3 卫星 240 8000 km 高度范围离子(不区分离子种类) 数据得到的上行离 子束事件通常发生在 4000 5000 km以上高度的结果 是比较一致的, 这是由于离子束的产生受到了大尺 度平行电场存在区域的限制. 对于离子雉事件, 可以 看到在 $2000 \mathrm{~km}$ 以上都有离子雉事件发生, 它们随高 度的分布相对离子束要均匀一些. 这和Gorney等人 ${ }^{[7]}$ 的结果也是一致的.

(ii) 发生率随 MLT 的变化. 离子束和离子雉 事件发生率随 MLT 的分布如图 8 所示.

低能离子束主要发生在 09:00 12:00 MLT扇区 和 21:00 00:00 MLT扇区, 高能离子束则主要发生 在 03:00 06:00 MLT扇区和午夜前扇区(21:00 MLT); 这与图 4 显示的总的 $\mathrm{O}^{+}$上行事件发生率随MLat和 MLT分布的图像是大体一致的. 我们得到的离子束 事件随MLT分布的特征和Kondo等人 ${ }^{[10]}$ 分析DE-1 卫 星 8000 23000 km高度范围数据得到的结果有一些 差别. Kondo得到的平静时低能离子束出现率几乎不 依赖于地方时, 高能离子束主要出现在夜晚, 磁午夜 前 (21:00 00:00 MLT)出现率最大. 对于离子雉事件, 从图 8 可以看到, 低能离子上行发生率在 09:00 12:00 MLT扇区占主导. 这和Gorney等人 ${ }^{[7]}$ 和 Kondo等人 0 7 7 - 得到的白天侧极尖/隙区是离子雉主 


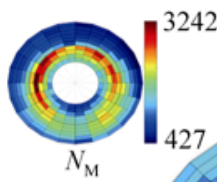

12

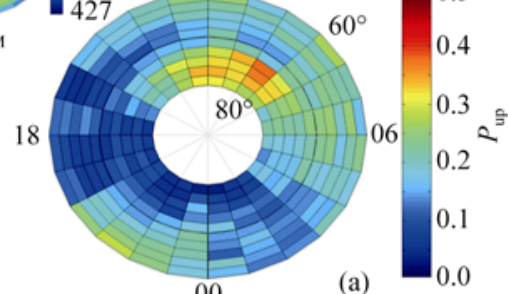

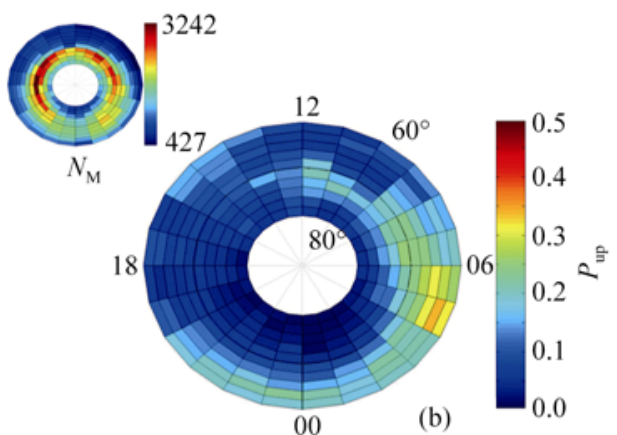

(b)

图 5 总的 $\mathrm{O}^{+}$上行事件发生率随 MLat 和 MLT 的分布

(a) 低能 $(E<1 \mathrm{keV}) \mathrm{O}^{+}$, (b) 高能 $(E>1 \mathrm{keV}) \mathrm{O}^{+}$. 每幅图左上角的小极图给出了每个网格里数据点总数
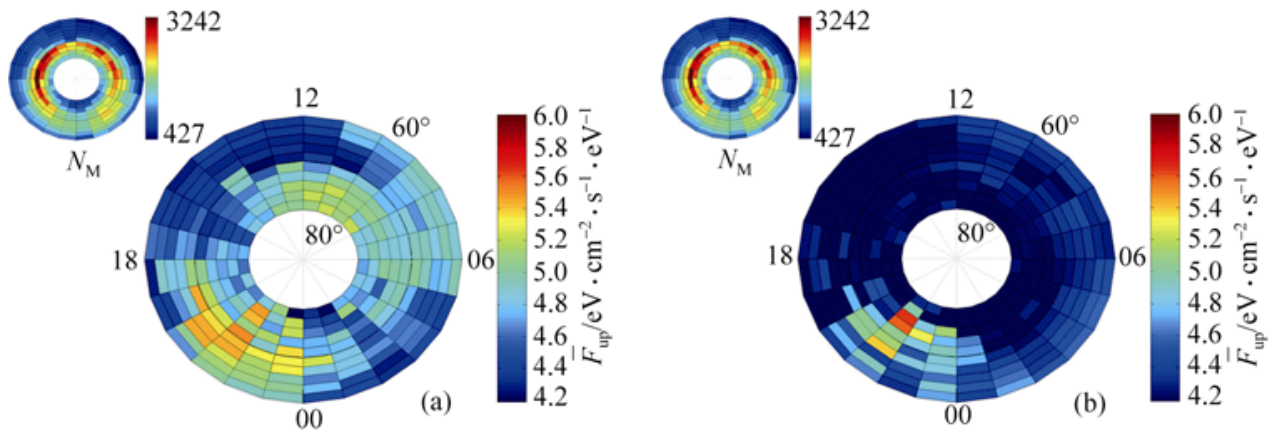

图 $6 \mathrm{O}^{+}$上行事件平均能通量随 MLat 和 MLT 的分布

(a) 低能 $(E<1 \mathrm{keV}) \mathrm{O}^{+}$; (b) 高能 $(E>1 \mathrm{keV}) \mathrm{O}^{+}$. 每幅图左上角的小极图给出每个网格里数据点总数
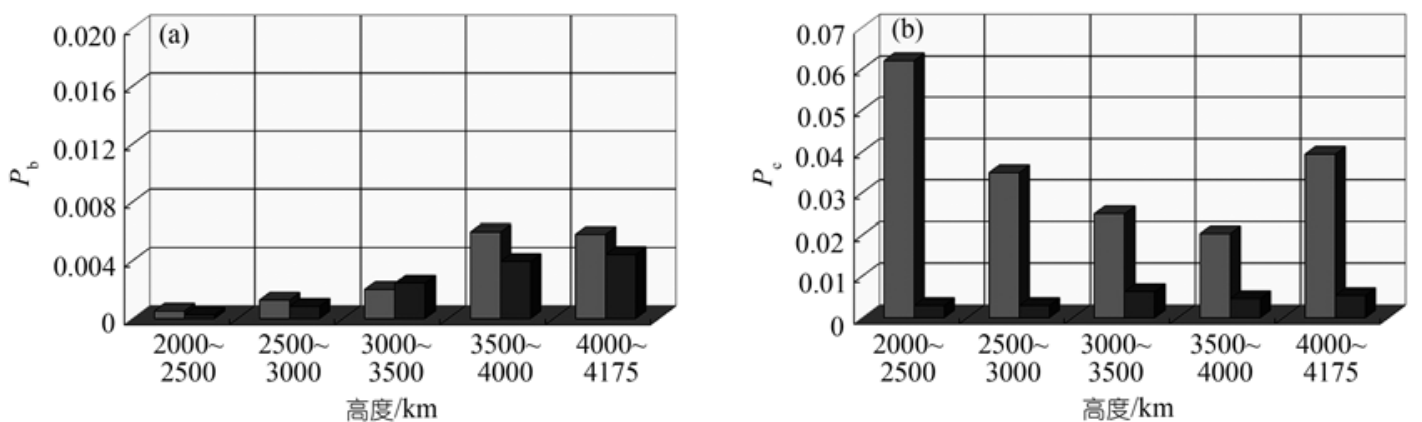

图 $7 \mathrm{O}^{+}$上行离子束 $(\mathrm{a})$ 和离子雉 $(\mathrm{b})$ 事件发生率随高度的分布

$\square E<1 \mathrm{keV}$,

- $E>1 \mathrm{keV}$

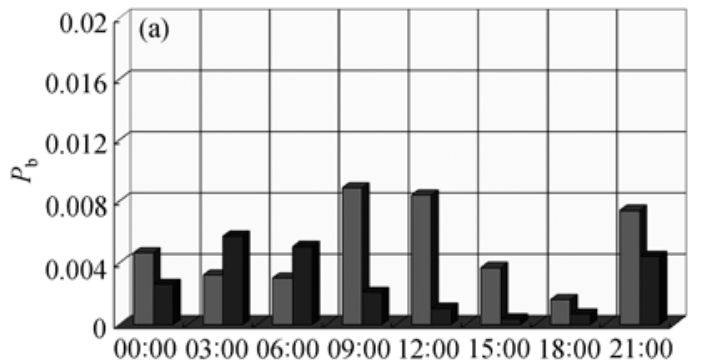

磁地方时

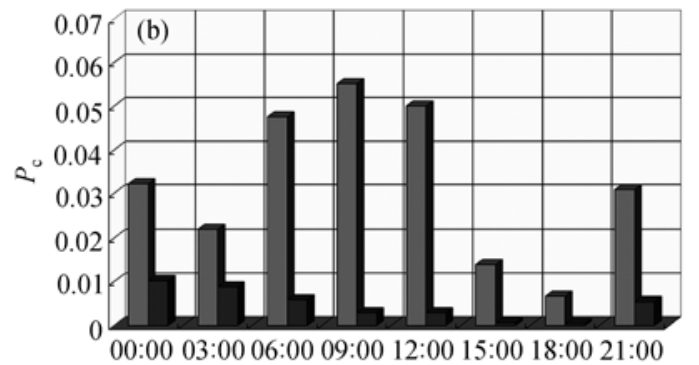

磁地方时

图 $8 \mathrm{O}^{+}$上行离子束(a)和离子雉 $(\mathrm{b})$ 事件发生率随 MLT 的分布

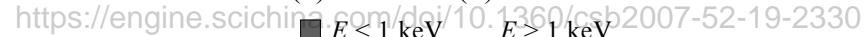


要发生区域的结果是比较一致的. 高能离子雉上行 主要发生在午夜及午夜后扇区.

(iii) 平均能通量随 MLT 的变化. 图 9 给出了 $\mathrm{O}^{+}$上行离子束/雉事件在所有高度和纬度上累加求平 均的能通量随磁地方时的变化. 可以看出, 低能离子 束在黄昏到午夜扇区 $(18: 00 \sim 00: 00$ MLT) 能通量最高, 09:00 MLT 附近扇区能通量次之; 高能离子束能通 量较大的区域主要是 15:00 MLT 扇区和 21:00 00:00 MLT 扇区. 低能离子雉能通量在各个地方时 分布比较均匀, 高能离子雉能通量较大值主要出现 在黄昏到午夜扇区.

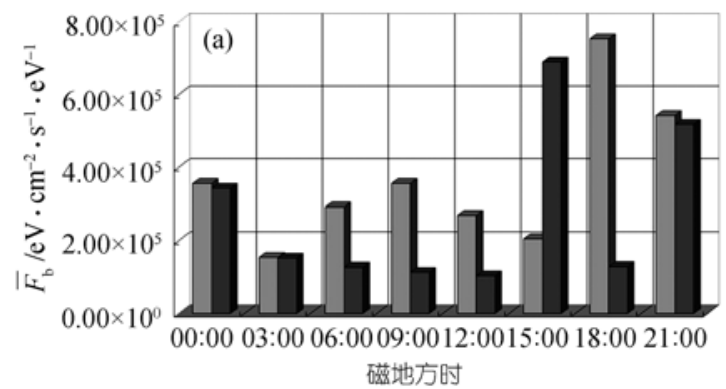

\section{3 讨论}

将前面得到的总的 $\mathrm{O}^{+}$上行发生率与平均能通量 随磁纬和磁地方时分布的图像与极区电离层电动力 学重要参量主要是极区等离子体对流以及极光椭圆 边界的静时平均分布图像作比较, 寻找平静时 $\mathrm{O}^{+}$离 子上行多发区与上行能通量最强区域的动力学特征.

\section{1 总的 $\mathrm{O}^{+}$上行与电子沉降之间的关系}

我们利用Feldstein极光椭圆模型 ${ }^{[27]}$, 计算 $K_{\mathrm{p}}=2$ 情况下椭圆的朝极和朝赤道边界坐标; 把极光椭圆 看作为电子沉降区域的表征. 图 10 给出极光椭圆边 界与总的 $\mathrm{O}^{+}$上行发生率及平均能通量随磁纬度和磁

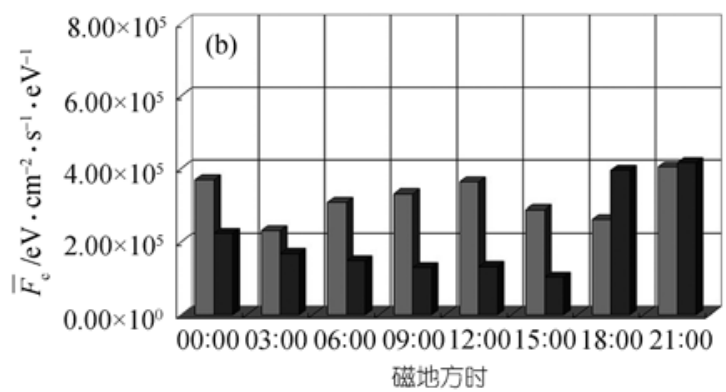

图 $9 \mathrm{O}^{+}$上行离子束 $(\mathrm{a})$ 和离子雉(b)平均能通量随 MLT 的分布

$\square E<1 \mathrm{keV}, \square E>1 \mathrm{keV}$
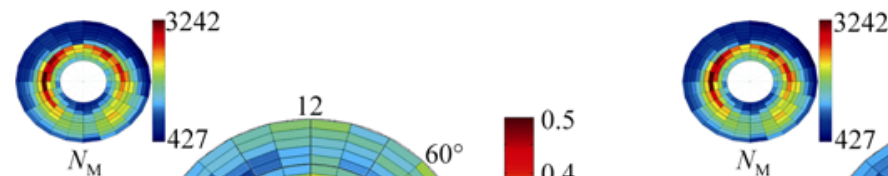

3242

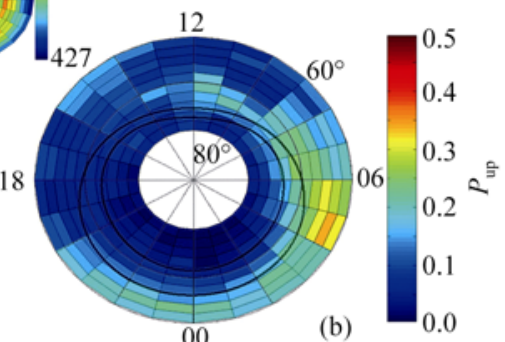

(a)
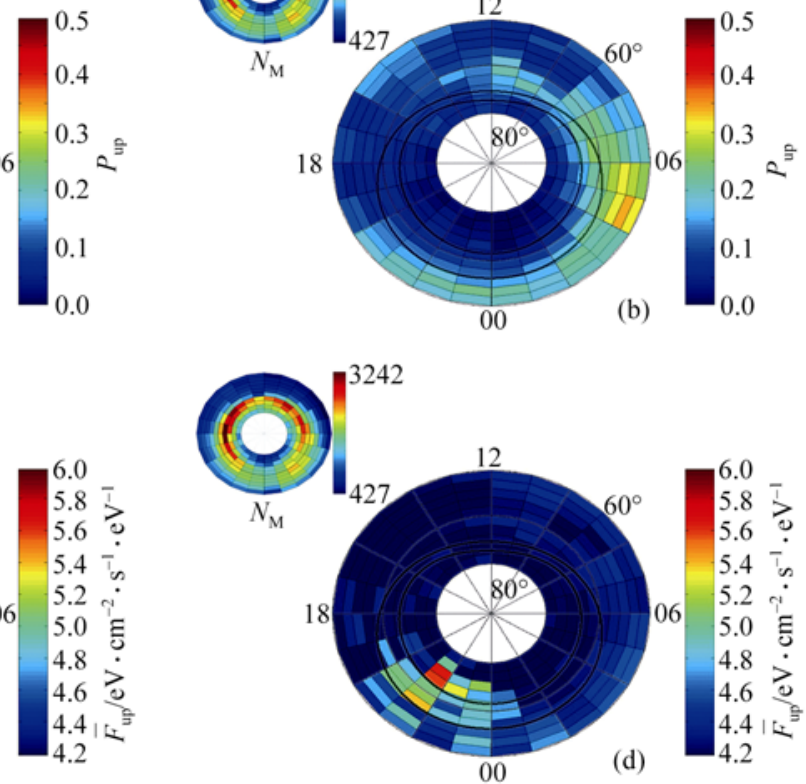

(c)

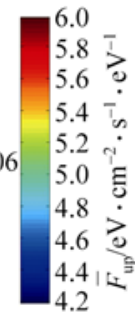

图 10

极光椭圆边界(黑粗线)与上行出现率 $(\mathrm{a}, \mathrm{b})$ 和平均能通量 $(\mathrm{c}, \mathrm{d})$ 分布的叠加, 其中 (a), (c) 两图对应的是低能 $(E<1 \mathrm{keV}) \mathrm{O}^{+}$离子, https://engir(b),(d)两图对应的是高能 $(E \mid>1 \mathrm{keV}) \mathrm{O}^{+}$离子-52-19-2330 
地方时分布叠加在一起的图像.

从图 10(a)可以清楚地看出, 低能 $\mathrm{O}^{+}$离子总的上 行发生率最大的区域位于磁正午前的极尖/极隙区, 处在极盖与极光椭圆分界附近. 同时, 上行平均能通 量最大区域也与黄昏后午夜前扇区极光椭圆带基本 相符(如图 10(c)所示). 这表明电离层离子上行与电 子沉降之间有着密切的联系. 近十年来的观测结果 ${ }^{[16,28]}$ 和数值模拟结果 ${ }^{[29,30]}$ 均表明, 极光电子沉降是 高纬 $\mathrm{F}$ 层/顶部电离层离子上行重要的驱动因素之一. 其中, 软电子 $(E<1 \mathrm{keV})$ 沉降对电离层离子上行有 更为直接的影响; 这些沉降电子通过加热电离层当 地的电子, 使得电子温度升高, 引起双极电场(与电 子温度成正比)的增强, 从而驱动离子上行.

对于高能 $\mathrm{O}^{+}$离子, 上行平均能通量最大区域与 黄昏后午夜前扇区极光椭圆带基本相符(图 10(d)), 但大部分上行事件出现在平静极光椭圆以外的更低 纬度上(图 10(b)). 这表明了电子沉降并不是驱动电 离层离子上行的唯一因素, 还存在其他控制因素.

\section{2 总的 $\mathrm{O}^{+}$上行与极区等离子体对流之间的关系}

我们利用Weimer模式 [31]计算极区电离层电位分 布，其等值线图即等离子体对流速度流线图; 计算时, 根据本文所用数据点年份和季节分布, 以及对应的
行星际磁场 $B_{y}$ 和 $B_{z}$ 分量取值分布情况, 取 $B_{y}=+2 \mathrm{nT}$, $B_{z}=-0.5 \mathrm{nT}$, 日期取为 1999 年春分点, 对应的太阳 日下点倾斜角 $(\mathrm{Tilt})$ 为 $-3^{\circ}$.

图 11 给出极区等离子体对流与 $\mathrm{O}^{+}$上行事件发生 率及平均能通量随磁纬和磁地方时分布叠加在一起 的图像.

从图 11(a)可以看到, 低能 $\mathrm{O}^{+}$离子总的上行发生 率最大的区域处在晨昏两个对流涡旋的转换区; 在 黎明至上午及磁午夜前 20:00 23:00 MLT 比较宽阔 的区域里出现的较显著的上行发生率, 处在高纬等 离子体对流区域内; 上行平均能通量最大区域位于 午夜前 21:00 23:00 MLT 扇区, 同时也是西向对流 速度比较大的区域或对流转向剪切较大的地方(如图 11(c)所示).

对于高能 $\mathrm{O}^{+}$离子, 最大上行发生率出现在 $04: 00$ 06:00 MLT 扇区较低纬度上, 处于晨侧一个相对孤 立的对流小涡旋的最大剪切区域(图 11(b)); 高能 $\mathrm{O}^{+}$ 靠近极盖边界处上行能通量最大的地方, 处在对流 从南向转为西向位置, 剪切相对较大(图 11(d)).

以上结果表明, 电离层离子上行与极区等离子 体对流之间有着非常紧密的联系. 对流能够从不同 的方面引起离子上行, 其中主要包括两个方面; 强对

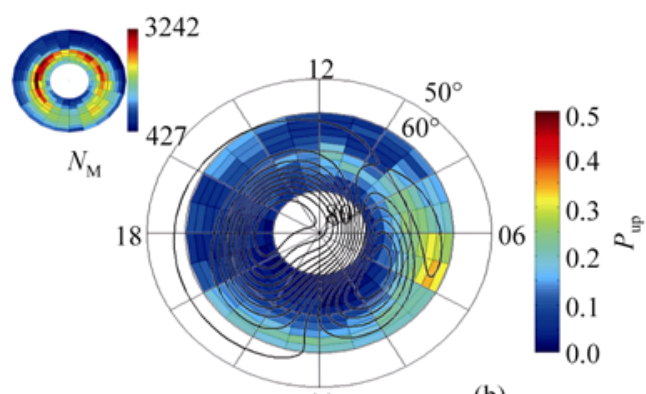

(a)
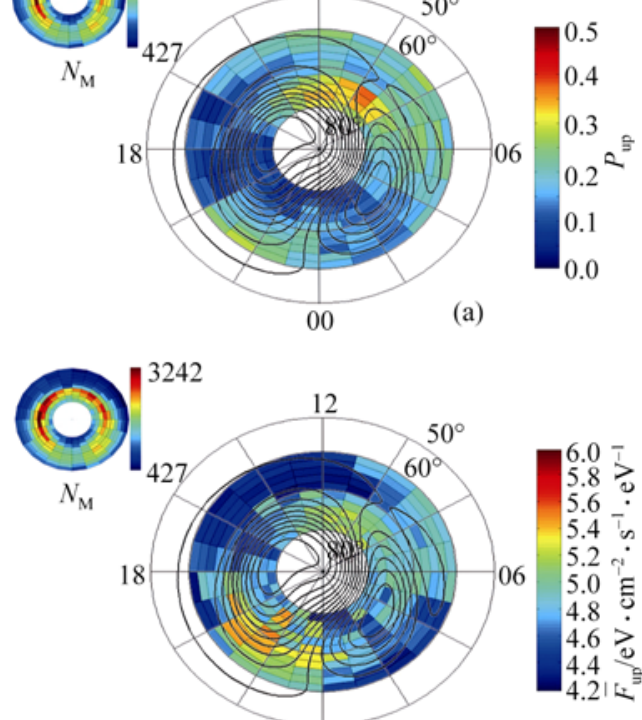

(c)

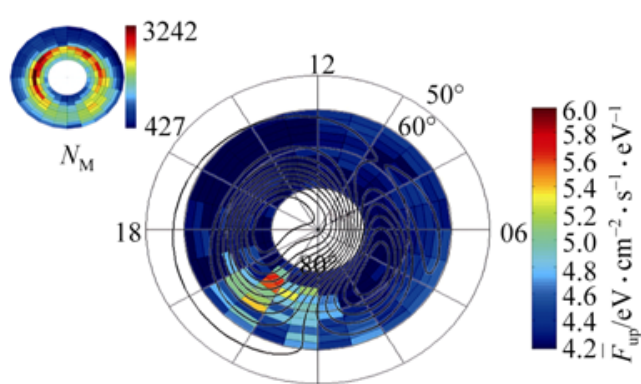

$00 \quad$ (d)

图 11

极区等离子体对流(黑粗线)与上行出现率 $(\mathrm{a}, \mathrm{b})$ 和平均能通量 $(\mathrm{c}, \mathrm{d})$ 分布的叠加, 其中 (a), (c)两图对应低能 $(E<1 \mathrm{keV}) \mathrm{O}^{+}$离子, https://engine (b), (d)两图对应高能 $(E>1 \mathrm{keV}) \mathrm{O}$ 离子 
流驱动离子上行和对流剪切驱动离子上行. 强对流 使得等离子体与中性大气碰撞加剧, 引起加热膨胀 而上行, 同时存在强对流与磁场方向快速变化时会 引起离子沿场的“离心加速”, [32], 而且场向加速度与 离子质量无关, 结果质量大的重离子, 比如 $\mathrm{O}^{+}$离子, 由此获得较多的能量. 对于对流剪切驱动离子上行 情况, 在行星际磁场南向时, 强对流剪切较多发生在 极光椭圆带晨昏两个对流涡旋转换区; 这时最大上 行区域所在处的对流电场很弱, 甚至局地为零. 也有 时对流方向保持不变, 但大小随空间急剧变化而出 现强剪切. 这种伴随强剪切而局地电场很弱的现象 在磁暴个例研究中曾有报道 ${ }^{[33,34]}$. 对流剪切通常可 以激发频率介于离子回旋频率和低混杂波频率之间 的等离子体波，这种波能十分有效地加热离子，从而 驱动离子上行 ${ }^{[35]}$. 部分上涌的离子能够到达更高的 高度, 通过横向获能或场向加速等形式进一步获得 加速/加热, 最终克服地球重力逃逸出去.

对于纬度较低的发生率较显著的 $\mathrm{O}^{+}$上行区域, 如磁地方时分别为 21:00 22:00 MLT和 04:00 05:00 MLT而修正磁纬 60\%附近区域, 利用T96 磁层 磁场模式 ${ }^{[23,24]}$ 并采用与本文所用数据相应的平静期 太阳风和行星际条件, 对上述区域的磁力线进行追 踪, 表明它们在赤道面内的地心距离 4 5 个地球半径, 即通常情况下的等离子体层顶区域; 这些亚极光区 电离层 $\mathrm{O}^{+}$离子上行事件与等离子体层边界层 ${ }^{[36]}$ 过程 之间的关系, 有待进一步研究.

\section{4 总结}

本文利用 1997 2003 年跨越半个多太阳黑子周 期期间 FAST 卫星的离子能量-投郑角-质谱仪 (TEAMS) 和离子静电分析仪(IESA) 测量数据, 对 $2000 \sim 4200 \mathrm{~km}$ 高度范围, 平静期 $\left(K_{\mathrm{p}}<3\right)$ 来自极区电 离层的氧离子 $\mathrm{O}^{+}$上行的时空分布特征进行了统计分 析, 取得有意义的新结果. 选用的平静期涵盖约 407 个磁静日, 数据点总数约 37 万个. 所分析的氧离子 $\mathrm{O}^{+}$上行事件, 包括总的离子上行、离子束和离子雉. 分析结果概括如下:

关于总的 $\mathrm{O}^{+}$上行:

(i ) 发生率. 磁正午前的极尖/极隙区是低能 $(E<1 \mathrm{keV}) \mathrm{O}^{+}$上行发生最频繁的区域; 靠近黎明前等 离子体对流的低纬边界区域是高能 $(E>1 \mathrm{keV}) \mathrm{O}^{+}$上 行发生率最高区. 无论能量高低, 在平静极光椭圆边
界之外的较低纬度宽阔时区上存在频繁的 $\mathrm{O}^{+}$上行. 从半球分布看, 低能 $\mathrm{O}^{+}$上行主要发生在白天半球, 高能 $\mathrm{O}^{+}$上行主要发生在夜晚半球; 无论能量高低, 黎明半球是上行主要发生区, 特别是高能 $\mathrm{O}^{+}$, 上行 发生率的晨昏不对称性非常显著.

(ii) 平均上行能通量. 无论是低能还是高能离 子, 午夜前扇区上行 $\mathrm{O}^{+}$携带着较强的能通量, 磁地 方时范围从 20:00 MLT 到 00:00 MLT, 纬度范围从 $64^{\circ} \mathrm{MLat}$ 到 $76^{\circ} \mathrm{MLat}$, 与午夜前极光椭圆带基本相符, 是西向对流速度比较大的区域; 最大的上行能通量 发生在 21:00 22:00 MLT 之间, 其中高能 $\mathrm{O}^{+}$离子在 $74^{\circ} \sim 76^{\circ} \mathrm{MLat}$ 出现非常强的上行能通量, 这里靠近极 盖边界, 而且对流从南向转为西向.

关于上行 $\mathrm{O}^{+}$离子束和离子雉:

(i ) 在 2000 4000 km 高度范围, $\mathrm{O}^{+}$离子雉事件 发生率显著高于离子束事件; 离子雉发生率在磁正 午前占主导, 离子束发生率随 MLT 的分布与总的 $\mathrm{O}^{+}$ 上行特征大体一致.

(ii) 离子束和离子雉发生率随高度分布特征有 极为显著的差别: 离子束主要发生在 $3500 \mathrm{~km}$ 高度以 上, 在 $3000 \mathrm{~km}$ 以下很少发现离子束事件; 而离子雉 在各高度都有发生, 分布也相对比较均匀.

（iii）低能离子束能通量在黄昏到午夜扇区最高, 与总的 $\mathrm{O}^{+}$上行能通量随 MLT 的分布特征大体一致; 高能离子束能通量最大的区域在 15:00 MLT 扇区, 其次是午夜前扇区. 低能离子雉能通量随地方时分 布比较均匀, 高能离子雉能通量较大值主要出现在 黄昏到午夜扇区.

致谢 感谢美国 NASA 哥达德空间飞行中心和加州大学伯 克利分校通过国际互联网提供 FAST 卫星数据以及 FAST 项目组为本研究提供高分辨率的数据. 感谢刘振兴院士对 本研究的关心与有益讨论, 以及 COSPAR-2006 期间美国 Yi-Jiun Su 博士和 Eric J. Lund 博士等对本文工作提出的有 益建议.

\section{参考文献}

1 Shelley E G, Johnson R G, Sharp R D. Satellite observations of energetic heavy ions during a geomagnetic storm. J Geophys Res, 1972, 77: 6104-6110

2 Shelley E G. Heavy ions in the magnetosphere. Space Sci Rev, 1979, 23: 465-497

3 Chappell C R, Giles B L, Moore T E, et al. The adequacy of the ionospheric source in supplying magnetospheric plasma. J Atmos Terr Phys, 2000, 62: 421-436[DOI]

4 Yau A W. Sources of ion outflow in the high latitude ionosphere. 
Space Sci Rev, 1997, 80: 1-25[DOI]

5 Daglis I A. The role of magnetosphere-ionosphere coupling in magnetic storm dynamics. In: Tsurutani B T, Gonzalez W D, Kamide Y, et al, eds. Magnetic Storms, Geophysical Monograph 98-Magnetic Storms. Washington, DC: AGU, 1997. 107-116

6 Klumpar D M. Transversely accelerated ions: An ionospheric source of hot magnetospheric ions. J Geophys Res, 1979, 84: 4229 $-4237$

7 Gorney D J, Clarke A, Croley D, et al. The distribution of ion beams and conics below $8000 \mathrm{~km}$. J Geophys Res, 1981, 86: 8389

8 Yau A W, Beckwith P H, Peterson W K, et al. Long-term (solar-cycle) and seasonal variations of upflowing ionospheric ion events at DE-1 altitudes. J Geophys Res, 1985, 90: 6395-6407

9 Yau A W, Shelley E G, Peterson W K, et al. Energetic auroral and polar ion outflow at DE-1 altitudes: Magnitude, composition, magnetic activity dependence and long-term variations. J Geophys Res, 1985, 90: 8417-8432

10 Kondo T, Whalen B A, Yau A W, et al. Statistical analysis of upflowing ion beams and conic distributions at DE-1 altitudes. J Geophys Res, 1990, 95: 12091-12102

11 Thelin B, Aparicio B, Lundin R. Observations of upflowing ionospheric ions in the mid-altitude cusp/cleft region with the Viking satellite. J Geophys Res, 1990, 95: 5931-5939

12 Fuselier S A, Ghielmetti A G, Moore T E, et al. Ion outflow observed by IMAGE: Implications for source regions and heating mechanisms. Geophys Res Lett, 2001, 28: 1163-1166[DOI]

13 Pfaff R, Carlson C, Watzin J, et al. An overview of the fast auroral snapshot (FAST) satellite. Space Sci Rev, 2001, 98: 1-32[DOI]

14 Möbius E, Tang L, Kistler L M, et al. Species dependent energies in upward directed ion beams over auroral arcs as observed with FAST TEAMS. Geophys Res Lett, 1998, 25: 2029-2032[DOI]

15 Lund E J, Möbius E, Klumpar D M, et al. Direct comparison of transverse ion acceleration mechanisms in the auroral region at solar minimum. J Geophys Res, 1999, 104: 22801-22805[DOI]

16 Strangeway R J, Ergun R E, Su Y J, et al. Factors controlling ionospheric outflows as observed at intermediate altitudes. J Geophys Res, 2005, 110(A03221): doi: 10.1029/2004JA010829

17 Wilson G R, Ober D M, Germany G A, et al. Nightside auroral zone and polar cap ion outflow as a function of substorm size and phase. $\mathrm{J}$ Geophys Res, 2004, 109(A02206): doi: 10.1029/2003JA009835

18 Andersson L, Peterson W K, McBryde K M. Dynamic coordinates for auroral ion outflow. J Geophys Res, 2004, 109(A08201): doi: 10.1029/2004JA010424

19 Andersson L, Peterson W K, McBryde K M. Estimates of the suprathermal $\mathrm{O}^{+}$outflow characteristic energy and relative location in the auroral oval. Geophys Res Lett, 2005, 32(L09104): doi: 10.1029/2004GL021434

20 McFadden J P, Carlson C W, Ergun R E, et al. Spatial structure and gradients of ion beams observed by FAST. Geophys Res Lett,
1998, 25: 2021-2024[DOI]

21 Klumpar D M, Möbius E, Kistler L M, et al. The time-of-flight energy, angle, mass spectrograph (TEAMS) experiment for FAST. Space Sci Rev, 2001, 98: 197-219

22 Carlson C W, Pfaff R F, Watzin J G. The fast auroral snapshot (FAST) mission. Geophys Res Lett, 1998, 25: 2013-2016[DOI]

23 Tsyganenko $\mathrm{N}$ A. A model of the near magnetosphere with a dawn-dusk asymmetry: 1. Mathematical structure. J Geophys Res, 2002, 107(A8): doi: 10.1029/2001JA000219

24 Tsyganenko $\mathrm{N}$ A. A model of the near magnetosphere with a dawn-dusk asymmetry: 2. Parameterization and fitting to observations. J Geophys Res, 2002, 107 (A8): doi: 10.1029/2001JA000220

25 Collin H L, Peterson W K, Lennartsson O W, et al. The seasonal variation of auroral ion beams. Geophys Res Lett, 1998, 25: 4071 -4074[DOI]

26 Janhunen P, Olsson A, Peterson W K. The occurrence frequency of upward ion beams in the auroral zone as a function of altitude using Polar/TIMAS and DE-1/EICS data. Ann Geophys, 2003, 21: 2059-2072

27 Feldstein Y I, Isaev S I, Lebedinsky A I. The phenomenology and morphology of aurorae. Ann IQSY, 1969, 4: 311-348

28 Seo Y, Caton R, Horwitz J L. Statistical relationship between high-latitude ionospheric F-region/topside upflows and their drivers: DE-2 observations. J Geophys Res, 1997, 102: 7493 7500 [DOI]

29 Liu C, Horwitz J L, Richards P G. Effects of convection ion heating and soft-electron precipitation on high-latitude F-region upflows. Geophys Res Lett, 1995, 22: 2713-2716[DOI]

30 Su Y J, Caton R G, Horwitz J L, et al. Systematic modeling of soft-electron precipitation effect on high-latitude $\mathrm{F}$ region and topside ionospheric upflows. J Geophys Res, 1999, 104: 153$163[\mathrm{DOI}]$

31 Weimer D R. An improved model of ionospheric electric potentials including substorm perturbations and application to the Geospace Environment Modeling November 24, 1996 event. J Geophys Res, 2001, 106: 407-416[DOI]

32 Horwitz J L, Moore T E. Four contemporary issues concerning ionospheric plasma flow to the magnetosphere. Space Sci Rev, 1997, 80: 49-76[DOI]

33 马淑英, 刘会欣, Schlegel K. 磁暴期间极光椭圆与极盖区电离 层效应比较研究一 F 区负暴. 中国地球物理学报, 2002, 45(2): $160-169$

34 Liu H, Lu G. Velocity shear-related ion upflow in the low-altitude ionosphere. Ann Geophys, 2004, 22: 1149-1153

35 Ganguli G, Keskinen M J, Romero H, et al. Coupling of microprocesses and macroprocesses due to velocity shear: An application to the low-altitude ionosphere. Geophys Res Lett, 1986, 13: $893-896$

36 Carpenter D, Lemaire J. The plasmasphere boundary layer. Ann Geophys, 2004, 22: 4291-4298 